\title{
Entwicklung eines neuartigen Magnetseilwindensystems - MAGWIN -
}

\author{
Dipl.-Ing. Rüdiger Herhold, Prof. Dr.-Ing. habil. Thorsten Schmidt \\ Technische Universität Dresden \\ Institut für Technische Logistik und Arbeitssysteme \\ Professur für Technische Logistik
}

\begin{abstract}
Basierend auf den Forschungen zum Verhalten der Elemente Seil, Rolle, Treibscheibe bzw. Trommelwinde in Seiltrieben in den unterschiedlichsten Einsatzfällen wurden in den letzten Jahren die Untersuchungen zur Erhöhung der Treibfähigkeit von Treibscheiben sowie die Abschätzung der Beanspruchung und der daraus resultierenden Schädigung und Lebensdauer der Elemente des Seiltriebs kontinuierlich fortgesetzt. In diesen Rahmen ordnet sich das Kooperationsprojekt „Neuartiges Magnetseilwindensystem -MAGWIN-“ ein.
\end{abstract}

Gegenstand der Untersuchungen sind neuartige Seilwinden. Sie stellen eine technische Anwendung der neuentwickelten mehrrilligen Magnettreibscheibe dar (vorgetragen auf dem 5. Fachkolloquium der WGTL 2009). Die prinzipielle Funktionsfähigkeit der Magnettreibscheibe für $F_{1} / F_{2}$ von 2 bis 3 wurde theoretisch und experimentell nachgewiesen. Die Erhöhung der Treibfähigkeit auf $F_{1} / F_{2} \sim 30$ bis 40 durch das Hintereinanderschalten von $x$-Scheiben in einem Windensystem und die damit erreichbaren Effekte sind Ziel dieser Arbeiten.

Daraus ergeben sich spezielle Herausforderungen, zu deren Lösung verschiedene Schritte geplant sind: die Entwicklung eines Magnetseilwindenprüfstandes und die Erarbeitung von Grundlagen zur Entwicklung neuer Produkte unter Einsatz der Magnettreibscheibe. Das Hauptaugenmerk liegt auf der Konzeptentwicklung und die Auslegung des Prüfstandes. Er wurde so konzipiert, dass sowohl verschiedene Systemvarianten für Scheibenanordnung und Antriebe (Prüfstand 1) als auch die Versuchsmuster für Havarie-Winden, Elektrozüge und Rangierwinden unter Betriebsbeanspruchungen (Prüfstand 2 bzw. Prüfstanderweiterung) getestet werden können. Für die zu entwickelnden Elektrozüge wird die Seilspeicherung in einem Seiltopf oder einer ähnlichen Konstruktion entwickelt und getestet. 


\section{Ausgangslage}

Die Forschung zum Verhalten von Seilen in Seiltrieben bei den unterschiedlichsten Einsatzfällen hat am Institut für Technische Logistik und Arbeitssysteme (TLA) eine lange Tradition. Schwerpunkte waren und sind dabei die Berechnung und Erhöhung der Treibfähigkeit von Treibscheiben und die Abschätzung der Seilschädigung.

Beim Antrieb von Aufzügen und Befahranlagen mit Seilen über Treibscheiben ist die Kraft $F_{1}$ an der auflaufenden Seite mit der Kraft $F_{2}$ an der ablaufenden Seite über die Eytelweinsche Gleichung

$$
\frac{F_{1}}{F_{2}}=e^{\mu \cdot \beta}
$$

- im Folgenden auch als Treibfähigkeit bezeichnet - verknüpft. Gl. (1) gilt für das biegeschlaffe Seil, in [Sch04] wird ihre Herleitung umfassend erläutert. Die maximal übertragbare Umfangskraft der Treibscheibe - sie ergibt sich aus der Differenz von $F_{1}$ und $F_{2}-$ wird durch die Parameter $\beta, \mu$ und $F_{2}$ bestimmt. Es kann bei der klassischen Treibscheibe nur erhöht werden, wenn mindestens ein Parameter vergrößert wird. Hier stößt man aber auf physikalisch bzw. technisch bedingte Grenzen. Für erforderliche Materialeinsparungen im Aufzugssystem (Senkung der Korbmasse $F_{2}$ ) und den Verzicht auf seilbeanspruchende Rillenformen (Reduzierung des Reibwertes $\mu$ ) bekommt der Einsatz von Neodym-Eisen-Bor-Magnete (NdFeB-Magnete) zur Steigerung der Treibfähigkeit eine große Bedeutung [Grä08]. Sie gehören zu den Seltene-Erden-Magneten und sind derzeit die leistungsstärksten Magnetwerkstoffe. Ihre extrem hohe Haftkraft, verbesserte Materialeigenschaften und die Entwicklung von preisgünstigen Beschichtungsverfahren haben dem Werkstoff weltweit zum Durchbruch verholfen.
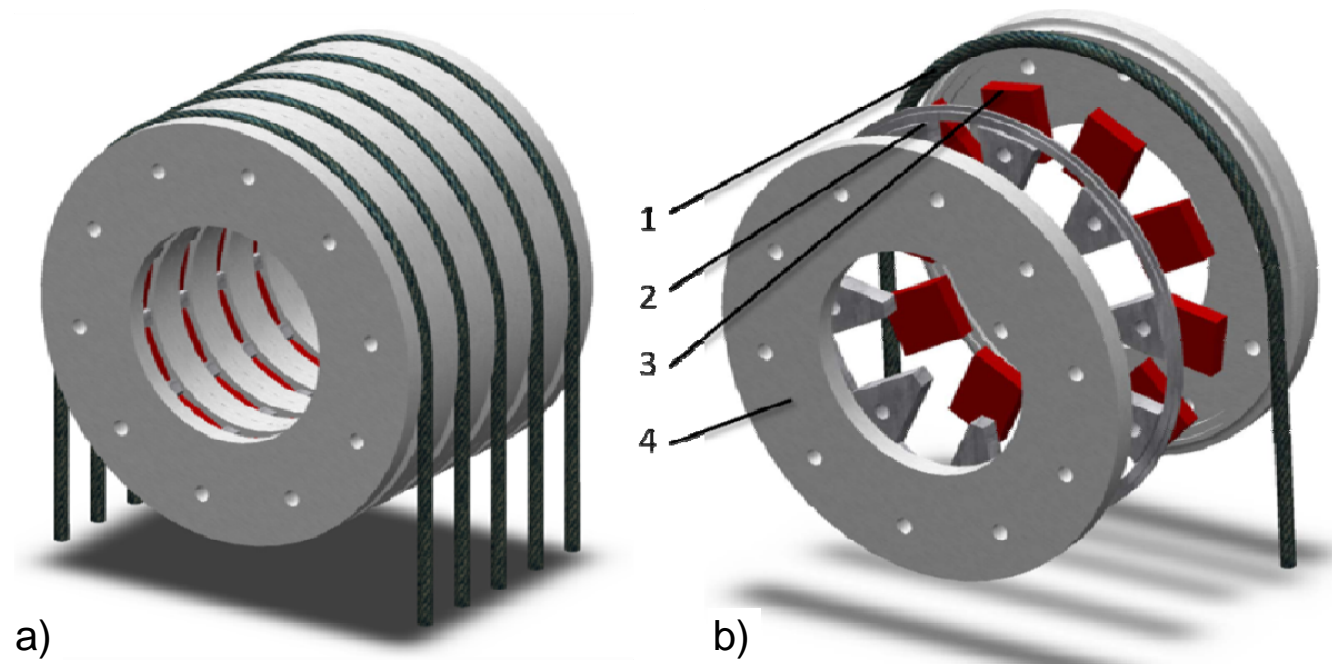

b)

Abbildung 1: Grundaufbau einer mehrrilligen Magnettreibscheibe

a) Treibscheibenpaket

b) Komponenten für eine Rille: 1 Seil, 2 Distanzscheibe,

3 Permanentmagneten, 4 Polscheiben 
Eine neue Lösung basiert im Ergebnis theoretischer Voruntersuchungen [Grä08] auf dem Einsatz von Hochleistungspermanentmagneten, die im Umfangsbereich von Treibscheiben konstruktiv angeordnet werden [Grä04]. Die Abbildung 1 zeigt den Grundaufbau am Beispiel einer fünfrilligen Magnettreibscheibe. Hier nimmt der geteilte Rillenkörper das Magnetsystem, bestehend aus Permanentmagneten und Distanzscheibe, auf. Zur Normalkraft infolge der Aufzugs- bzw. Gegengewichtsmasse kommt die Magnetkraftwirkung hinzu. Durch den Einsatz solcher Hochleistungspermanentmagnete im Umfangsbereich einer Treibscheibe konnte die Treibfähigkeit signifikant erhöht und dauerhaft garantiert werden.

Gegenstand der aktuell laufenden industriellen Forschung sind neuartige Seilwinden. Sie stellen eine technische Anwendung der neuentwickelten mehrrilligen Magnettreibscheibe dar (vorgetragen auf dem 5. Fachkolloquium der WGTL 2009). Die prinzipielle Funktionsfähigkeit der mehrrilligen Magnettreibscheibe und ihre erhöhte Treibfähigkeit wurden theoretisch und experimentell nachgewiesen. Für das Gebiet der Seilwinden sind nun nachhaltige Lösungen erforderlich. Es gilt, Materialressourcen durch neue Entwicklungen effizienter zu nutzen, um so die Umwelt zu entlasten. Im vorliegenden Vorhaben sind dafür $u$. a. Grundlagen für den industriellen Einsatz neuer Materialien zu schaffen. Durch die Kooperationspartner sollen im Rahmen dieses SAB-Projektes Lösungen erbracht werden, die diesem Ziel dienen.

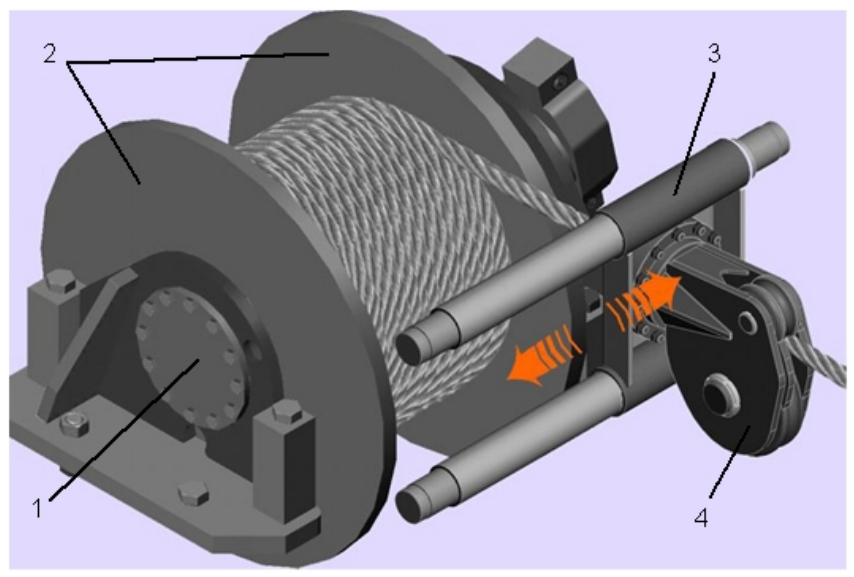

1 - Trommelmantel

2 - Bordscheiben

3 - Spuleinrichtung

4 - Schlaffseilsicherung

Abbildung 2: Windentrommel vom Typ Spulmat [Feuerwehrvortrag Celle]

Die bekannteste Bauform von Seilwinden ist die Windentrommel. Eine klassische Bauart der Windentrommel ist in Abbildung 2 dargestellt. Sie wird häufig als Hubwinde in Kranen verwendet, aber auch in Standard-, Hub- und Bergegeräten stellt sie das zugkrafterzeugende Maschinenteil dar [Hen00]. Da der Trommelkörper zugleich den Seilspeicher des Zugseiles darstellt, kommt es bei Mehrlagenwicklungen zu erhebliche Quetschungen des Seiles, Reduzierung der Seillebensdauer und Ausfallzeiten durch Seilwechsel [Wei08]. Der Einsatz des Trommelkörpers als Antriebstrommel macht eine erheblich stabilere Auslegung der Trommel (Stahl) erforderlich. Hier setzt der Einsatz der Magnettreibscheibe vor allem an - der konsequente Leichtbau wird möglich. 


\section{Entwicklung des Magnetseilwindenprüfstandes}

\subsection{Grundprinzip und Zielstellung}

Die prinzipielle Funktionsfähigkeit der Magnettreibscheibe (MTS) für $F_{1} / F_{2}$ von 2 bis 3 wurde in vorherigen Untersuchungen theoretisch und experimentell nachgewiesen. Die Erhöhung der Treibfähigkeit auf $F_{1} / F_{2} \sim 30$ bis 40 durch das Hintereinanderschalten von $x$-Scheiben in einem Windensystem und die damit erreichbaren Effekte sind Ziel dieser Untersuchungen. Die Magnettreibscheibe eröffnet dabei vollkommen neue technische Lösungen im Windenbau. Um große Umfangskräfte übertragen zu können, sind im Vergleich zum Stand der Technik keine Keilrillen und Anpressvorrichtungen erforderlich, sondern materialschonende Rundrillen mit kleinem Unterschnitt und Permanentmagneten.

Die Entwicklung von Magnettreibscheiben für Seilwinden mit bedeutend verbesserten Parametern der Magnettreibscheibe und die Erarbeitung von Grundlagen zur Entwicklung neuer Produkte unter Einsatz dieser neuen Magnettreibscheibe sollen in diesem Kooperationsprojekt gelöst werden. Dazu wurde ein Windenprüfstand (Prüfstand 1) entwickelt, der in der Entwicklungsphase der Magnetseilwinden die Testung der unterschiedlichen Windenkonzepte ermöglicht und in der Versuchshalle des Institutes errichtet wird. In Zusammenarbeit mit den Kooperationspartnern werden experimentelle Untersuchungen zur Anordnung von Einzeltreibscheiben durchgeführt. Die Grundstruktur für ein Dimensionierungsverfahren von in Reihe bzw. parallel geschalteter Einzelmagnettreibscheiben in Abhängigkeit von unterschiedlichsten Randbedingungen wird entwickelt und für zukünftige Produkte und Systeme nutzbar gemacht. Dies betrifft vor allem Randbedingungen wie die Magnetauslegung je Einzelscheibe, die Kombination von Magnettreibscheiben mit losen Rollen, Kosten und Platzbedarf. Auf einer Prüfstanderweiterung (Prüfstand 2) werden die durch die Partner entwickelten Versuchsmuster getestet und bewertet.

Im Ergebnis dieser gesetzten Zielstellungen wurde ein Prüfstandskonzept entwickelt, das folgenden Anforderungen und Aufgaben entsprechen soll:

- Nachweis der Treibfähigkeit und Seilkraftreduzierung für $x$ Scheiben

- Testung des Verhaltens im Langzeitbetrieb

- Untersuchung der Treibscheibenabnutzung

- Schlupfverhalten bei mehrrilligen Treibscheiben oder mehrstufigen Anordnungen

- Auswirkung des Verschleißes auf das Treibverhalten

- Ermitteln kompakter Anordnungen

- Messen von Seilkraftverläufen zwischen den einzelnen Stufen

- Test verschiedener Anordnung treibender und getriebener Scheiben

\subsection{Seilführungen}

Die Seilführungen ermöglichen grundsätzlich verschiedene Versuchsaufbauten. Das Seil kann in unterschiedlichen Varianten über ein- und mehrrillige Magnettreibschei- 
ben verlegt werden. So wurden mögliche Scheibenkombinationen für Versuche am Prüfstand 1 umfassend analysiert. Dabei galt es, die möglichen Positionen der Seilkraftmessungen zu berücksichtigen und zu ermöglichen (zwischen den beiden Treibscheibenreihen bzw. vor und hinter den beiden Seiltrommeln), die Summe der Umschließungswinkel zu variieren und die Anbindung der beiden Seiltrommel zu gestalten. Daraus resultierend wurden zwei Hauptseilführungen mit folgenden Charakteristika festgelegt:

- Beim einfachen Überlaufen jeder Treibscheibe (Abbildung 3 A) ist ein Seilkraftmessen vor und hinter jeder Scheibe möglich

- Beim mehrfachen Überlaufen der beiden mehrrilligen Magnettreibscheiben (Abbildung 3 B) kann beliebig nach der Scheibe 2 bzw. vor der Scheibe 1 gemessen werden

Wahlweise können einzelne Treibscheiben auch antriebslose geschalten werden und so als einfache Umlenkrolle fungieren. Hauptsächlich verändern sich dabei die Umschlingungswinkel der Seile über den getriebenen Scheiben.
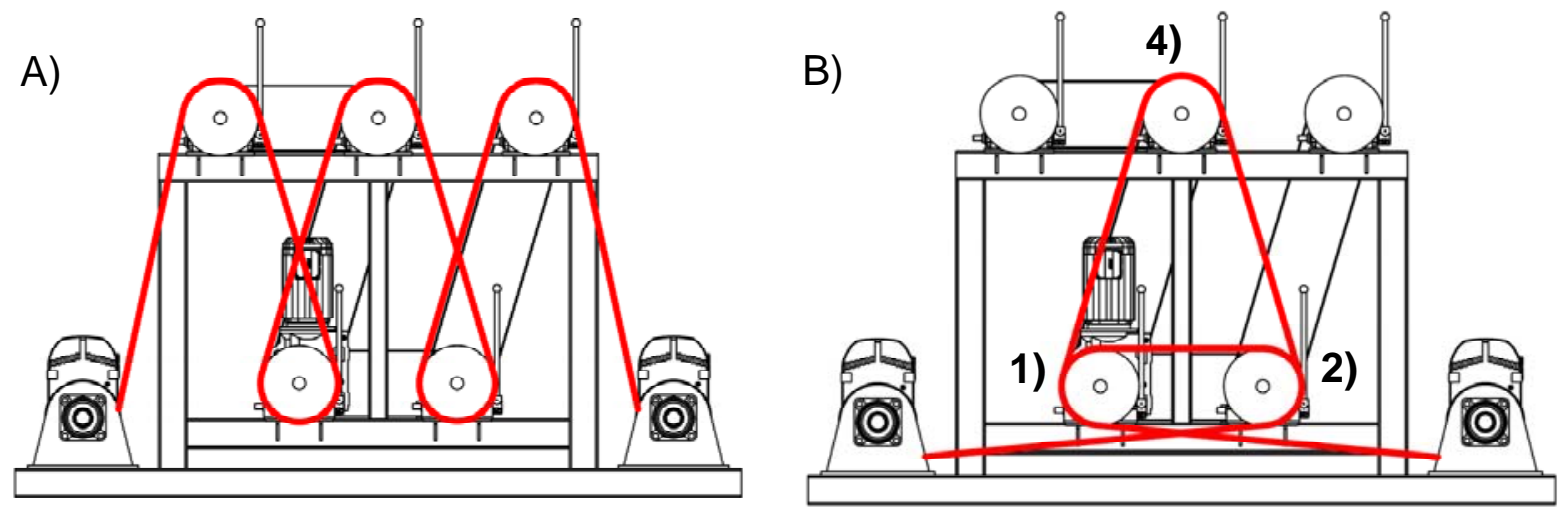

Abbildung 3: Hauptseilführungen A (mit fünf einrilligen MTS) und B (zwei dreirillige MTS)

Die maximal mögliche Grenztreibfähigkeit an einer mehrrilligen Magnettreibscheibe mit Halbrundrillen und Unterschnitt kann mit der dafür entwickelten erweiterten Eulergleichung nach $\mathrm{Gl}$. (2) berechnet werden.

$$
\frac{F_{1}}{F_{2}} \text { Grenz }=e^{\mu \beta}+\frac{q_{M} \cdot \frac{d}{2} \cdot \pi \cdot\left(1-\frac{\alpha^{\circ}}{180^{\circ}}\right) \cdot \frac{D}{2}\left(e^{\mu \beta}-1\right)}{\frac{F_{2}}{i \cdot z}}
$$

Dabei bedeuten:

$$
\begin{array}{ll}
\frac{F_{1}}{F_{2}} \text { Grenz } & \text { Maximale Treibfähigkeit der Magnettreibscheibe } \\
\text { d: } & \text { Seildurchmesser in } \mathrm{cm} \\
\text { D: } & \text { Treibscheibendurchmesser in } \mathrm{cm} \\
\mathrm{q}_{\mathrm{M}}: & \text { Magnetische Flächenpressung in } \mathrm{N} / \mathrm{cm}^{2}
\end{array}
$$


i:

Untersetzung bei indirekter Aufhängung

Z:

Seilzahl

Zur Auslegung der Treibscheibenanordnungen und der Dimensionierung des Versuchsstandes konnten mit dieser Berechnungsgrundlage (Gl. 9) mehrere Berechnungstabellen erstellt (Tabelle 1) werden. Mit deren Hilfe wurden die erwarteten Seilkraftentwicklungen simuliert und die Anzahl erforderlicher Umschlingungen (Summe der Umschließungswinkel) errechnet. Dies erfolgte für unterschiedliche Versuchsanordnungen, deren divergierende Parameter über variable Stellgrößen anpassbar sind (Tabelle 1 zeigt dies für den Seildurchmesser $10 \mathrm{~mm}$ unter Einsatz zweier dreirilliger Magnettreibscheiben mit Durchmesser $300 \mathrm{~mm}$ ). Das Ziel besteht darin, mit ausreichend vielen Umschlingungen die Seilkraft auf nahezu Null zu fahren.

Tabelle 1: Berechnungstabelle der Seilkraftentwicklung für Hauptseilführung B1

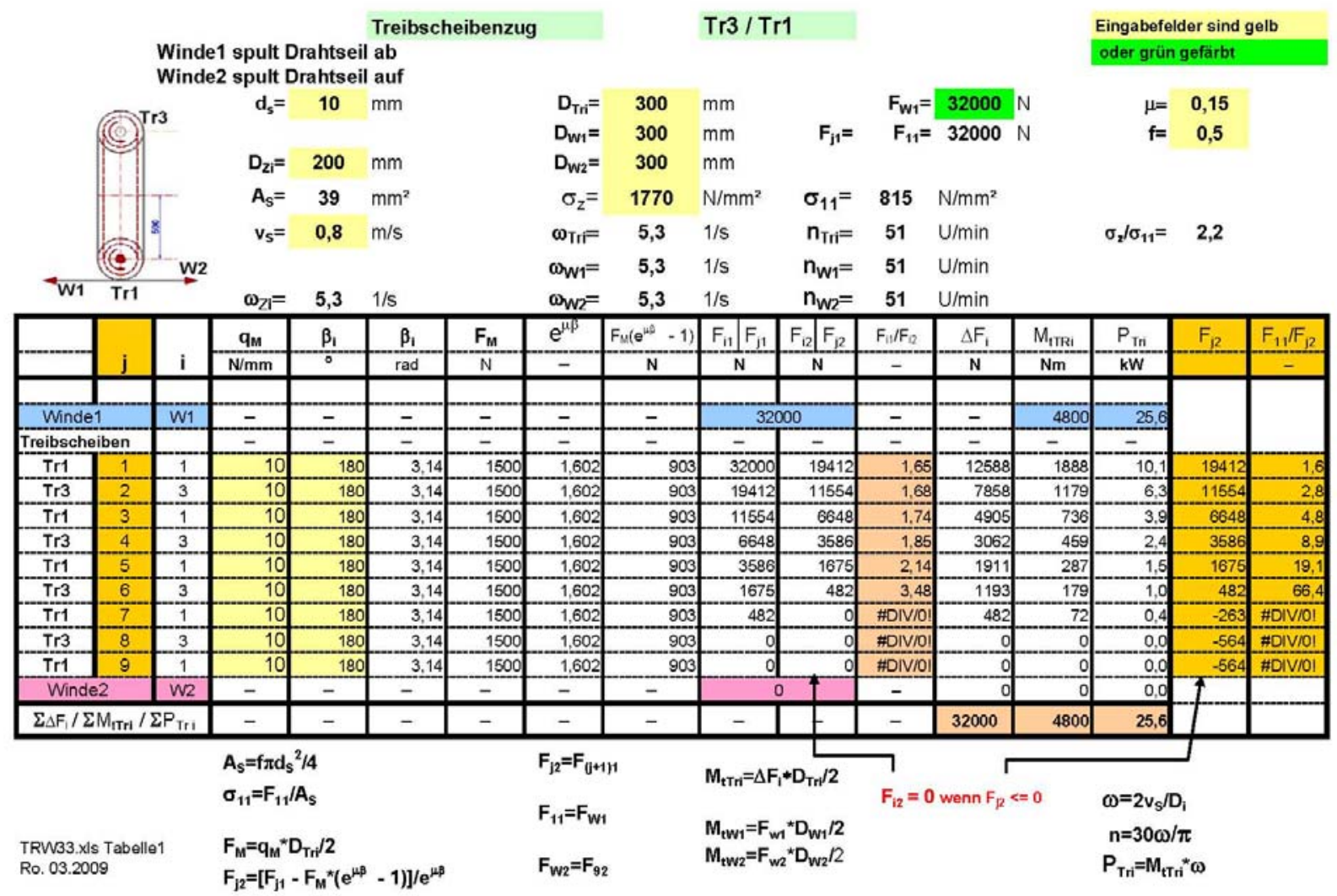

Auf den Erkenntnissen dieser Seilführungssimulationen aufbauend ist begonnen worden, die Geometrie der Magnetwindenprüfstände abzuleiten.

\subsection{Konzept des Prüfstandes}

Zur Untersuchung der unterschiedlichen Treibscheibenkombinationen und Testprodukte ist die Erzeugung einer regelbaren Seilzugkraft erforderlich. Zu deren Aufbringung wurden das Gegenmasseprinzip, eine Hydraulikzylindervariante und das Win- 
denprinzip untersucht. Im Ergebnis eines binären Variantenvergleiches wurde das Windenprinzip mit drehmomentgeregelten Lastwinden ausgewählt. Vor allem die Variabilität und kompakte Bauweise ermöglichen ein breites Untersuchungsspektrum. Für die unterschiedlichen Versuchsanordnungen, schnellen Seilkraftänderungen und die Vielfalt der Testprodukte (modulare Erweiterbarkeit) bieten sich gute Prüfbedingungen. Das Ergebnis zeigt Abbildung 4. Für jede Treibscheibenaufnahme ist eine Kupplung vorgesehen, um ein wahlweises Kraftlosschalten jeder Scheibe zu ermöglichen.

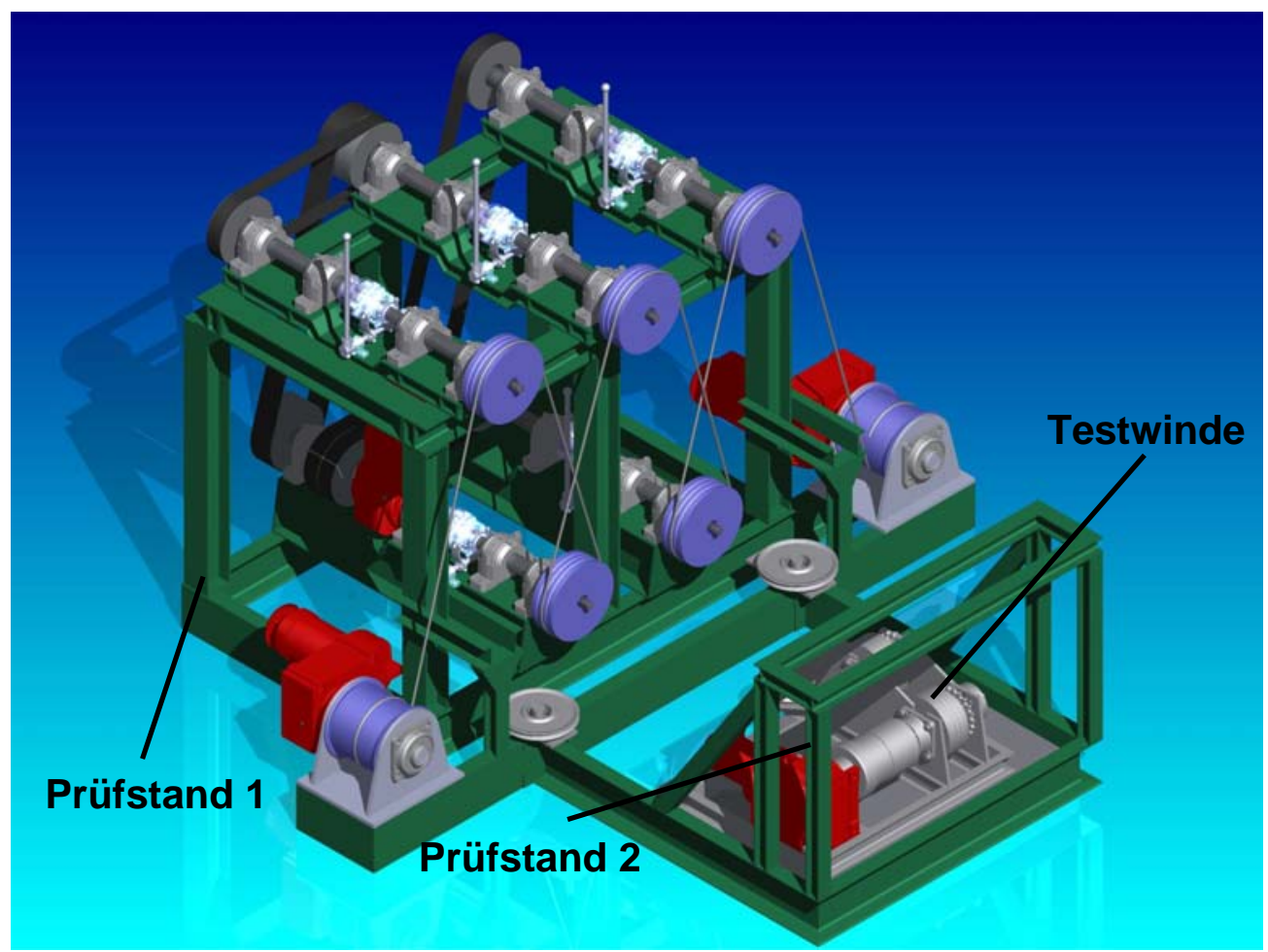

Abbildung 4: Magnetseilwindenprüfstände mit Testwinde

\subsection{Leistungsdaten und Betriebsarten des Prüfstandes}

Die Leistungsdaten des Prüfstands werden durch einige wenige Parameter beschrieben. Zentral sind hierbei die aufzubringende Ausgangsseilkraft $F_{s o}$ sowie die maximale Prüfdauer je Durchlauf, in Abhängigkeit der Seilgeschwindigkeit $v_{s}$. Die maximale Ausgangsseilkraft, die sich an den zu testenden Windensystemen ausrichtet, wurde mit $26 \mathrm{kN}$ festgelegt. Da die Treibscheibendurchmesser je nach Prüfseil austauschbar und variabel ausgelegt werden müssen, besteht zusätzlich die Anforderung an eine variable Drehzahlsteuerung. Dazu zeigt Tabelle 2 die Momentenmatrix, welche im Überblick einen sehr großen Regelbereich darstellt. Basierend auf dem Seildurchmesser wird die Seilkraft über den Sicherheitsfaktor $5<v<7$ bestimmt und auf volle $\mathrm{kN}$ gerundet. 
Tabelle 2: Momentenmatrix

\begin{tabular}{|c|c|c|c|c|}
\hline $\mathrm{F}_{\mathrm{s} 0}$ & $\mathrm{~d}_{\mathrm{s}}$ & \multicolumn{3}{|c|}{ Nennmomente } \\
\hline$[\mathrm{N}]$ & {$[\mathrm{mm}]$} & $\mathrm{D}_{\mathrm{s}}=240 \mathrm{~mm}$ & $\mathrm{D}_{\mathrm{s}}=360 \mathrm{~mm}$ & $\mathrm{D}_{\mathrm{s}}=480 \mathrm{~mm}$ \\
\hline 4.000 & 6 & $480 \mathrm{Nm}$ & $720 \mathrm{Nm}$ & $960 \mathrm{Nm}$ \\
\hline 8.000 & 8 & $960 \mathrm{Nm}$ & $1.440 \mathrm{Nm}$ & $1.920 \mathrm{Nm}$ \\
\hline 16.000 & 12 & $1.968 \mathrm{Nm}$ & $2.880 \mathrm{Nm}$ & $3.840 \mathrm{Nm}$ \\
\hline 26.000 & 16 & $3.259 \mathrm{Nm}$ & $4.710 \mathrm{Nm}$ & $6.226 \mathrm{Nm}$ \\
\hline
\end{tabular}

Bei den errechneten Momenten für Treibscheiben mit dem Durchmesser $D_{S}=240 \mathrm{~mm}$ sowie $D_{S}=360 \mathrm{~mm}$ bei einem Seildurchmesser $d_{S}=16 \mathrm{~mm}$ handelt es sich nur um theoretische Werte, da das in der Fördertechnik übliche Verhältnis $D_{S} / d_{S}=30$ weit unterschritten wird. Der auszulegende Drehzahlbereich des Prüfstands ergibt sich demnach aus den zu fahrenden Seilgeschwindigkeiten $v_{s}$ von 0,3 bis $1,2 \mathrm{~m} / \mathrm{s}$ und bewegt sich zwischen 12 und 95 1/min.

Zur Gewährleistung des umfangreichen Prüfprogramms sind für die Ansteuerung der zwei Winden- und des einen Treibscheibenantriebes folgenden Betriebsarten und Anforderungen festgelegt worden:

- Tippbetrieb, Dauerlauf, Dauerlauf mit n-Lastwechseln

- Anfangsdrehrichtung manuell wählbar

- Seilrisserkennung (plötzlicher Kraftabfall), ggf. Rollrad mit Taster

Die Seilkraft wird zu Beginn entweder im Einrichtbetrieb oder durch Eingabe eines Sollwertes eingestellt. Diese Seilkraft muss auch bei Stillstand und Richtungswechsel erhalten bleiben. Eine Winde dient als Last und die andere Winde trommelt das Seil je nach Drehrichtung der Treibscheiben auf oder ab. Die Drehzahl der Treibscheibe ist variabel.

\section{$3 \quad$ Seilspeicher für ein belastungsfreies Seil}

\subsection{Anforderungen an den Seilspeicher}

Der Seilspeicher hat die Aufgabe, das belastungsfreie Seil einer Seilwinde zu speichern. Dafür sind bestimmte Anforderungen notwendig, um die wirtschaftlichste Wickelvariante des Seilspeichers zu ermitteln. Der Speichervorgang soll ohne zusätzlichen motorischen Antrieb geschehen, nur abhängig von der Schubkraft des Seiles sein. Das spart Energie, Bauraum und nicht zuletzt Kosten, indem viele Bauelemente wie Getriebe, Rutschkupplung etc. entfallen. Durch den Eigenantrieb des Seiles soll sich das Seil in seine günstigste, geordnete Position legen, um das Befüllen wie auch die Entnahme des Seiles aus dem Speicher so leichtgängig und unkompliziert wie möglich zu gestalten. Das erhöht die Lebensdauer des Seiles durch verringerten Verschleiß. Des Weiteren soll die Neigung des Seilspeichers variiert werden, um das (C) 2010 Logistics Journal : Proceedings - ISSN 2192-9084 
Wickelverhalten unter verschiedenen Positionen zu untersuchen. Dabei ist ein Winkel bis zu $90^{\circ}$ von Interesse. Größere Winkel sind nicht sinnvoll, da ansonsten ein Aufwickeln entgegen der Gewichtskraft stattfindet. Es werden verschiedene Seilspeichergeometrien untersucht, um die wirtschaftlichste Variante zu ermitteln.

\subsection{Vorversuche}

In Vorversuchen sind erste Untersuchungen zum Wickelverhalten des belastungsfreien Seiles im Seilspeicher unternommen worden. Der Speichertopf gestaltete sich ähnlich der in Abbildung 5 dargestellten Konstruktionsvariante. Es zeigte sich, dass allein durch die Schubkraft des Seiles der Seilspeicher sich zu drehen beginnt. Jedoch sind gute Wickelbedingungen an einige Randbedingungen geknüpft.

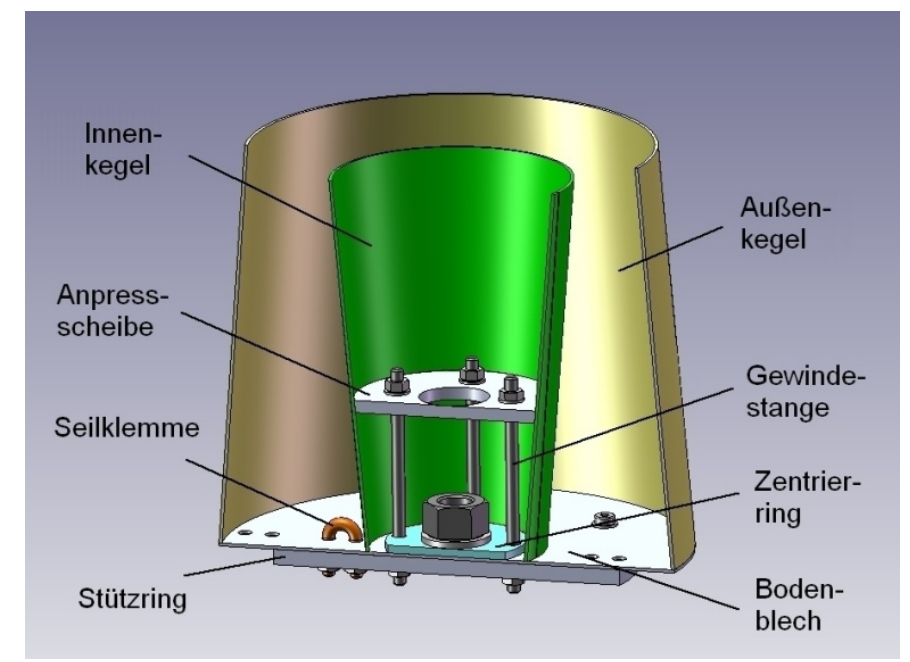

Abbildung 5: Konstruktionsvariante - kegeliger Seilspeicher

So erwies sich die Befestigung des Seiles tangential zum Bodenblech als sehr positiv, da die Schubkraft in direkter Drehrichtung eingeleitet wurde.

Eine besondere Aufmerksamkeit wurde der Seilführung geschenkt. Unter verschiedenen Einlaufwinkeln und Abständen wurde das Seil durch ein gerades Rohr in den Seilspeicher geführt. Dabei brachte ein kurzer Abstand der Seilführung zu der jeweiligen Seillage eine schnelle Reaktion in Form von Rotation des Seilspeichers. Ein Aufwärtsgleiten der Seilschlaufen an der Seitenwand konnte durch die konische Form vermieden werden.

Als kritisch hat sich der Seilauszug ergeben. Dabei brachten die unterschiedlichen Stellungen der Seilführung keine maßgeblichen Veränderungen, der kontrollierte Seilauszug ist vorrangig von der Beschaffenheit des Trommelkerns abhängig. Ein verwendeter Rundstab wies einen viel zu geringen Durchmesser auf, um das erforderliche Drehmoment für das Drehen des Seilspeichers erzeugen zu können. Es empfiehlt sich ein möglichst großer Durchmesser des Trommelkerns. 


\subsection{Versuchsaufbau Seilspeicher}

Der Versuchsaufbau für den Seilspeicher muss letztlich so gestaltet werden, dass er die eingangs genannten Anforderungen erfüllt und verschiedene zu untersuchende Konstruktionsvarianten enthält. Der konzipierte Versuchstand (Abbildung 6) bietet die Möglichkeit, dass immer zwei Varianten zur gleichen Zeit untersucht werden können. Über eine Kurbel, die den Seilantrieb realisiert, wird das Seil aus dem einen Seilspeicher entnommen und dem anderen wieder zugeführt.

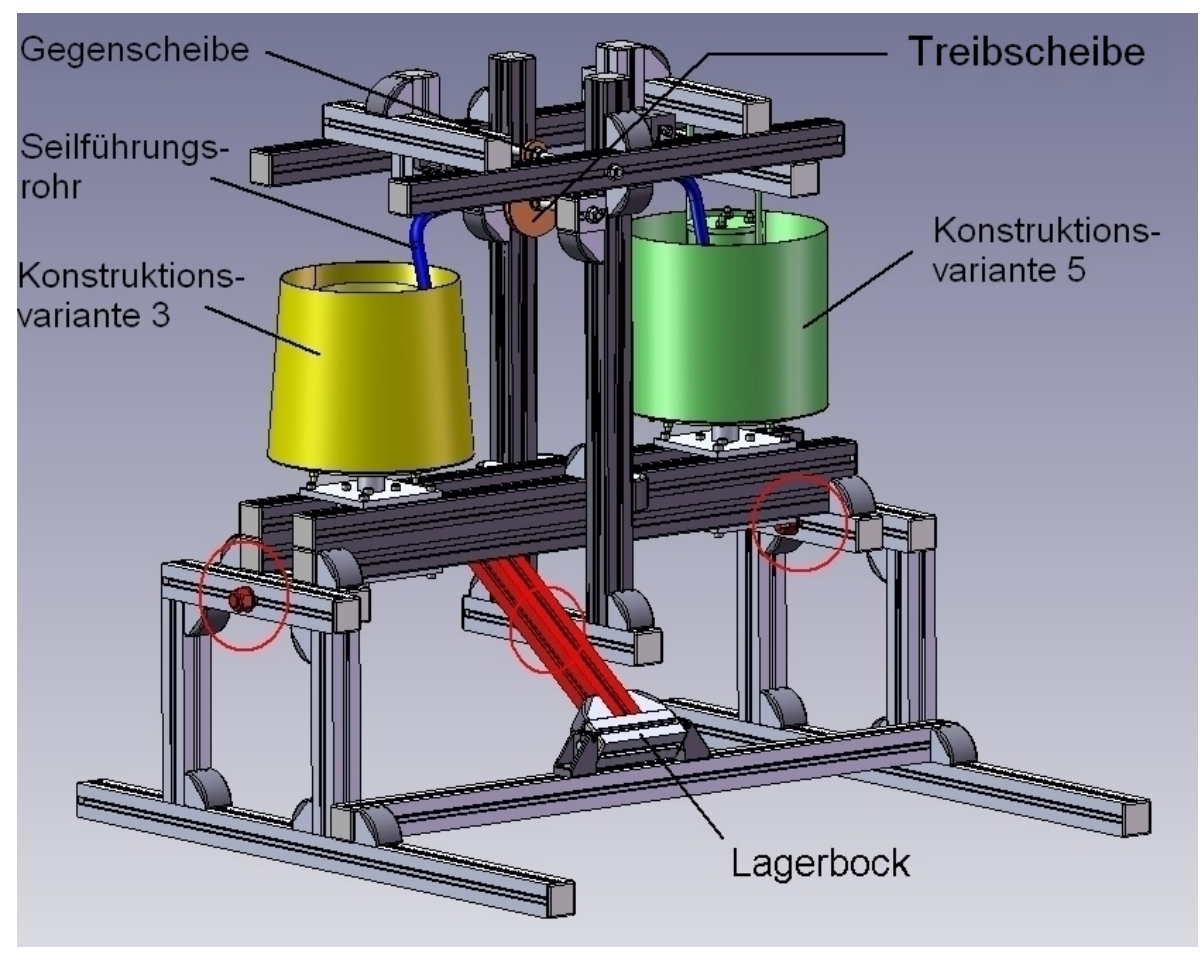

Abbildung 6: Versuchsaufbau Seilspeicher in vertikaler Lage

Um die Vorzugsvarianten untersuchen zu können, besitzen die Seilspeicher einen modularen Aufbau. Die Trommelkerne sind untereinander austauschbar und erhöhen somit die Untersuchungsvielfalt. Als weitere Bedingung für den Versuchsaufbau ist eine einstellbare Neigung gefordert. Die Aufnahme der Seilspeicher ist über eine Art „Drei-Punkt-Aufhängung“ konstruiert, die in Abbildung 6 rot markiert ist. Die Neigung des Prüfstandes um $90^{\circ}$ ist möglich.

Die Untersuchungen am Seilspeicher-Prüfstand dienen der Entwicklung einer Lösungsvariante, die in Verbindung mit einem Elektrozug eines Industriepartners zu einem umsetzbaren Produkt führen soll, deren Testung ebenfalls auf dem Magnetwindenprüfstand vorgesehen ist. Der Variantenentwicklung ist eine umfangreiche Patentrecherche vorausgegangen, um abzusehende Überschneidungen im vornherein zu vermeiden. 


\section{$4 \quad$ Zusammenfassung und Ausblick}

Der Beitrag stellt das Entwicklungskonzept eines Magnetseilwindenprüfstandes vor. Ziel des Prüfstandes ist es, die neuentwickelte mehrrillige Magnettreibscheibe für unterschiedlichste Windensysteme anwendbar zu machen. Dafür sollen sowohl verschiedene Systemvarianten für Scheibenanordnungen und Antriebe als auch Versuchsmuster untersucht werden. Seilverschleißende Spill- und Windentrommeln sollen durch gewichtsparende Seilspeicher aus dem Leichtbau ersetzt werden können. Dies ermöglicht erhebliche Materialeinsparungen bei Seileinsatz und Antriebssystemen. Erste Untersuchungen an Seilspeichertöpfen für belastungsfreie Seile für den Einsatz an Hubwinden wurden vorgestellt.

Der im Aufbau befindliche Prüfstand zur Untersuchung von Scheibenanordnungen kann modular erweitert und bei zukünftigen Forschungen für optimierte Treibscheibenkonstruktionen und Seilarten (vor allem hinsichtlich neuer Einsatzfelder) verwendet werden.

\section{Literatur}

[Grä04] Gräbner, Peter: Treibscheibe für Hochleistungsreibpaarungen, Europäische Patentanmeldung. Aktenzeichen AP 03720 161.3-1022, Aug. 2004.

[Grä08] Gräbner, Peter: Neue Wege bei der Anwendung des Leichtbaus in der Aufzugstechnik. In: Hebezeuge Fördermittel - Fachzeitschrift für Technische Logistik, Berlin 48 (2008) 5., S.356-359.

[Hen00] Henschel, J.: Dimensionierung von Windentrommeln. Düsseldorf: Vertriebsgesellschaft $\mathrm{mbH}, 2000$.

[SCH94] Scheffler, Martin: Grundlagen der Fördertechnik - Elemente und Triebwerke. Wiesbaden: Vieweg \& Sohn Verlagsgesellschaft,1994.

[Wei08] Weiskopf, U.: Untersuchung zur Lebensdauer von Kranhubwerken in der Mehrlagenwicklung. Stuttgart: Universität Stuttgart - Institut für Fördertechnik und Logistik, Dissertation, Juli 2008. 